\title{
UNA ESCALERA SUSPENDIDA
}

ALBERT SAMPER BLAS HERRERA
PROFESOR, UNIDAD PREDEPARTAMENTAL DE ARQUITECTURA, UNIVERSITAT ROVIRA I VIRGILI, TARRAGONA, ESPAÑA. PROFESOR, DEPARTAMENTO DE INGENIERÍA INFORMÁTICA Y MATEMÁTICAS, UNIVERSITAT ROVIRA I VIRGILI, TARRAGONA, ESPAÑA.

\section{FIG 1}

Escalera helicoidal del vestíbulo de acceso de la General Motors Technical Center. Warren, Michigan, 1956. Arquitecto / Architect: Eero Saarinen. Spiral staircase of the entrance lobby of the General Motors Technical Center. Warren, Michigan, 1956. http://www.pdparchitects.com/projects/ images/www_gmtech_r\&d-stair.jpg

\section{FIG 2}

Escalera helicoidal del Pabellón de

Canadá en la Expo de Bruselas de 1958. Arquitecto Charles Greenberg.

Spiral staircase of the Canadian Pavilion in the Brussels Expo of 1958. Architect Charles Greenberg. Fuente / Source: PRACHT, Klaus. Escaleras interiores y exteriores. Barcelona, Gustavo Gili, 1991 p. 37. Gentileza de American Iron and Stee Institute, Nueva York./ Courtesy of the American Iron and Steel Institute, New York

\section{FIG 3}

Escalera de cristal de la Tienda Joseph de la arquitecta Eva Jiricna en 1989, Londres. Fuente / Source: Eva Jiricna Architects, Londres.

Crystal staircase of the Joseph store by architect Eva Jiricna in 1989, London.

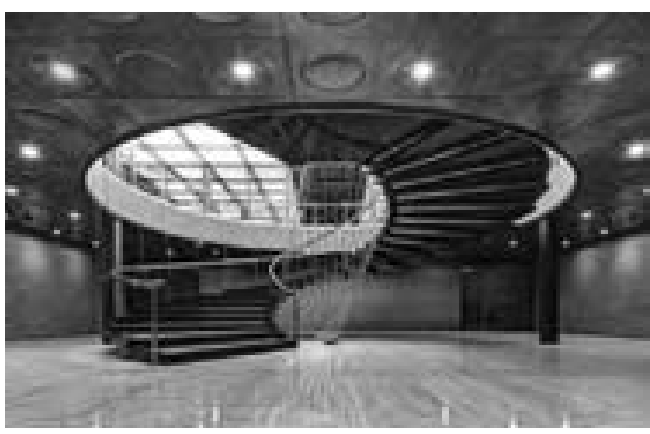

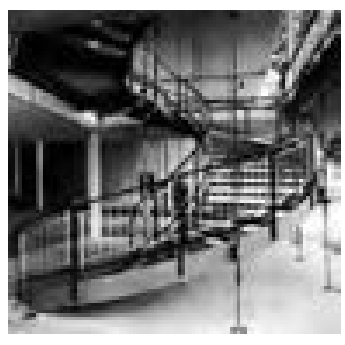

FIG 2

\section{A ESCALERA HELICOIDAL DEL HOTEL ROYAL SAS, ARNE JACOBSEN}

L as primeras escaleras colgadas se remontan a principios del siglo pasado. Arquitectos punteros como Rudolf Schindler o Richard Neutra empezaron a colocar escaleras suspendidas lineales en viviendas unifamiliares: los primeros ensayos consistían en suspender por un extremo cada uno de los peldaños y engastarlos por el otro lado. Las escaleras suspendidas empezaron a despuntar en 1935, cuando el arquitecto Frank Lloyd Wright diseñó una escalera lineal colgada en la Casa de la Cascada (Mc Carter, 1994 y Zabalbeascoa, 1998). El sistema constructivo de esta escalera era parcialmente similar al sistema planteado por Schindler y Neutra, ya que consistía en colgar íntegramente cada uno de los peldaños de tal manera que una barra atravesara dos peldaños para contrarrestar las vibraciones y el momento de torsión.

Tras la Segunda Guerra Mundial -y con la influencia de las soluciones tecnológicas y constructivas norteamericanas difundidas en publicaciones como Progressive Architecture- el sistema constructivo en acero se sofisticó y ello permitió aplicar nuevas fórmulas estructurales en el diseño de escaleras. Arne Jacobsen fue uno de los primeros arquitectos que
FIG 1

El edificio se construyó en la ciudad de $\mathbf{1}$ Warren, Michigan, entre 1949 y 1955 y fue inaugurado con la presencia del presidente de EE.UU. Dwight D. Eisenhower en 1956

El pabellón fue diseñado por los arquitectos 2 canadienses Harold Semmens y Douglas Simpson, y una vez finalizada la exposición fue vendido y reinstalado en la ciudad belga de Genk, donde sirve hasta la actualidad como la sede de una escuela primaria. adoptó las posibilidades que permitía el acero: un ejemplo de esta actitud es el diseño íntegramente suspendido de la escalera helicoidal, del vestíbulo del Hotel Royal sAs en Copenhague, cuyo proyecto fue desarrollado entre 1956 y 1961 . Otra escalera helicoidal con rasgos técnicos y constructivos muy parecidos a aquella diseñada por Jacobsen -y contemporánea a ella-fue la que el arquitecto Eero Saarinen construyó en el vestíbulo de acceso de la General Motors Technical Center ${ }^{1}$, (fig. 1) (Long y Peatross, 2008; Pelkonen y Albrecht, 2011). Un tercer caso que encarnó estos nuevos desarrollos fue la escalera helicoidal del Pabellón de Canadá ${ }^{2}$ (fig. 2) (Hoffmann y Griese, 1970) una de las tantas muestras de nuevos conceptos constructivos diseñados en acero presentadas en la Exposición Universal de Bruselas de 1958.

Tanto los diseños ejecutados por Arne Jacobsen y aquellos presentados en la Exposición Universal confirman la repercusión técnica y voluntad de innovación en el diseño de escaleras colgadas. Otro ejemplo claro es la escalera helicoidal diseñada por los arquitectos Friedrich Wilhelm, Günter Pfenning y Ernst Sieverts para el vestíbulo del Edificio de Administración de la ciudad de Brunswick, en 1959. En la actualidad, son muchos los ejemplos de escaleras suspendidas que tras un progreso constructivo han llegado a alcanzar esa voluntad de querer ocultar, en la medida de lo posible, cualquier tipo de detalle constructivo con el fin de hacer levitar este elemento de comunicación (Slessor, 2001 y Sánchez, 1990). Un caso representativo de este tipo de diseños es la escalera de la tienda Joseph (fig. 3) de la arquitecta Eva Jiricna, construida en 1989 en Londres (Jiricna, 1990 y 2001).

Por estos motivos, la escalera del Hotel Royal sAs merece un estudio personalizado. Pretendemos definirla mediante los espacios y los elementos del edificio que están proyectados en torno a ella, tratando de describir la intención de cada uno de los gestos de esta escalera hasta descubrir todas las capas que conforman el detalle constructivo.

Nuestro objetivo principal es revelar cada detalle y material. Pretendemos hacer una reconstrucción bidimensional y tridimensional tratando de aclarar documentación gráfica histórica que no describe con claridad este elemento. Y con todo, explicaremos la razón formal de diseñar una escalera suspendida en el vestíbulo principal del Hotel Royal SAs.

\section{MATERIAL Y MÉTODO}

Para abarcar la investigación, la principal fuente de información han sido las revistas de época Edilizia Moderna y Techniques et Architecture. Con la información general que hemos encontrado en estos registros hemos podido generar los documentos gráficos que relacionan la escalera con la arquitectura y diseño del edificio. Existe una gran variedad de monografías de Arne Jacobsen. Sin embargo, pocas son del nivel necesario para poder ser consideradas como punto de referencia en la presente investigación. La que más información ofrece, y en la cual se pueden apreciar los detalles más técnicos, es la monografía de Clásicos del Diseño de Arne Jacobsen (Solaguren, 1991).

En cuanto a la abundancia de información que ofrecen las fuentes de Internet, es preciso aclarar 
La escalera del hall del Hotel Royal SAS de Jacobsen es testimonio de una temprana exploración sobre elementos helicoidales suspendidos; ella aparece como un elemento puntual capaz de informar el proyecto completo.

PALABRAS CLAVE: ARQUITECTURA - DINAMARCA, ROYAL SAS, ARNE JACOBSEN, ESTRUCTURAS COLGANTES, HELICOIDE.
The stair at the Royal SAS Hotel hall shows the early exploration that Jacobsen did on suspended helicoids; it appears as a singular element whose design proved to influence the entire project.

KEYWORDS: ARCHITECTURE - DENMARK, ROYAL SAS, ARNE JACOBSEN, HANGING STRUCTURES, HELICOID. English text in p. 52
FIG 4

Hotel Royal SAS, Copenhague. Corte longitudinal.

Hotel Royal SAS, Copenhagen. Long section.

Escala / Scale. 1: 1.000

Trazos volumétricos pautan el programa; relación de la escalera con los diferentes niveles. Volumentric strokes outline the program; relationship of the stair with the different levels.

1. Instalaciones/sauna / Installations/ sauna

2. Suites / Suites

3. Habitaciones / Rooms

4. Nivel de personal / Personnel leve

5. Administración / Administration

6. Restaurante / Restaurant

7. Vestíbulo / Lobby

8. Bar / Bar

9. Tienda / Store

10. Hotel lobby / Hotel lobby

11. Patio de invierno / Winter patio

12. Snack bar

13. Sótano de servicio / Service basement

14 Estacionamiento / Parking

15. Bar

16 Vestíbulo de pasajeros / Passenger

lobby

17. Agencia de viajes / Travel agency

18. Comedor de personal / Personnel dining room

\section{FIG 5}

Hotel Royal SAS, Copenhague. Planta general, nivel $+5,18$.

Escala / Scale. 1: 1.000.

Hotel Royal SAS, Copenhagen. General Plan, level $+5,18$.

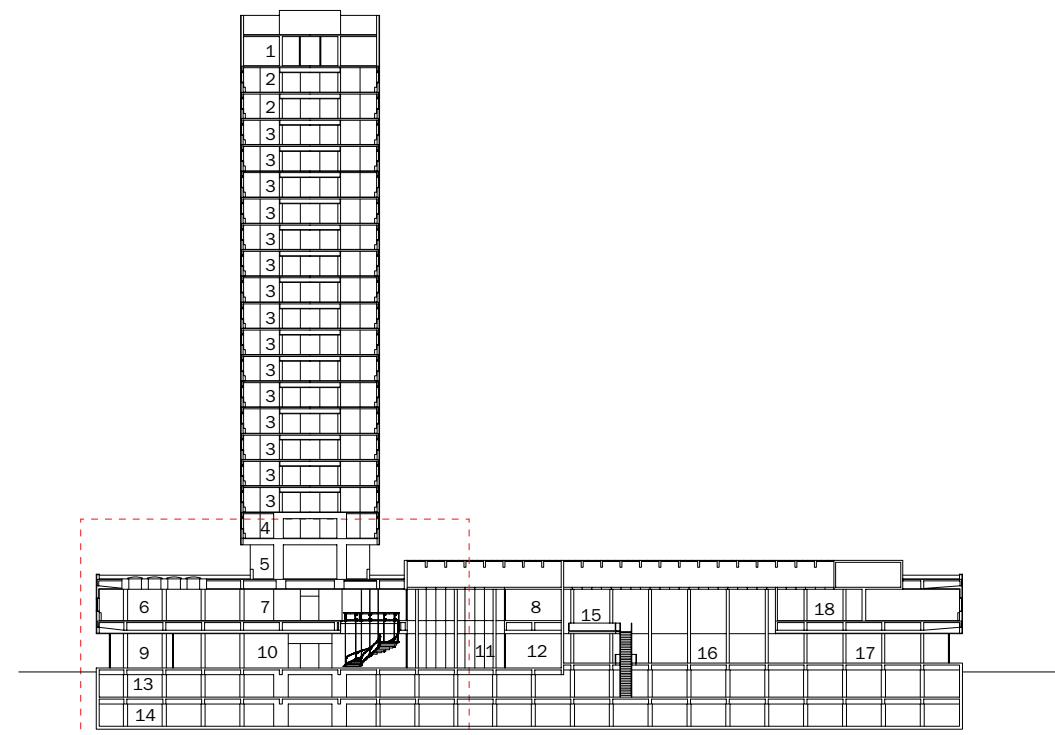

FIG 4

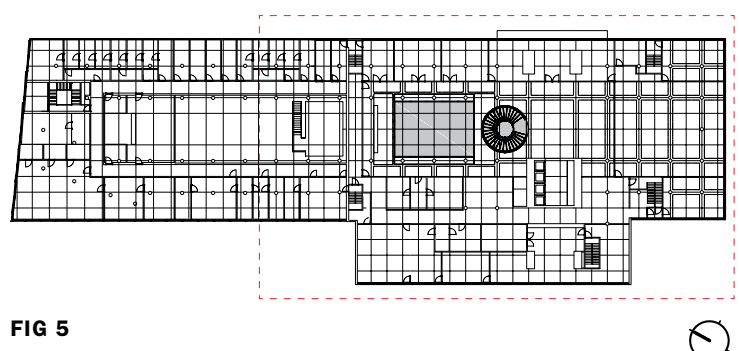

que debe ser contrastada y tratada posteriormente para mejorar el enfoque de los conceptos. Buscadores especializados como Google Académico, la Biblioteca del Colegio Oficial de Arquitectos de Cataluña, el Catálogo de la Universitat Politécnica de Catalunya o la base de datos de la Royal Institute British Architects, han sido las principales herramientas de indagación por su rigurosidad en la información y su proximidad.

Para poder llevar a cabo el presente trabajo, el cual requiere nuevos puntos de vista, hemos reelaborado todos los documentos originales con la rigurosidad necesaria para poder generar uno nuevo y hemos creado nuevos modelos tridimensionales. El documento está dotado de las cualidades oportunas para transmitir los rasgos de estudio. El proceso de reconstrucción se ha hecho mediante la observación de documentos gráficos, ya sean fotografías o planos técnicos. Posteriormente, la informatización se ha realizado a través de programas como AutoCad, en el caso de hacer una reconstrucción bidimensional, y de Google Sketchup, en el caso de hacer una reconstrucción tridimensional.
El proceso empieza compilando la mejor información posible para disponer de una descripción exhaustiva que permita observar la globalidad del proyecto y afrontar el análisis. Seguimos con una posterior digitalización de calidad de todos los documentos, planos o fotografías. Los nuevos dibujos los hemos delineado guardando fidelidad al original, pero con nuevos valores de trazos de dibujo, aportando el valor de línea y las tramas adecuadas para poner de relieve las circunstancias que hemos querido destacar. Basándonos en estos nuevos dibujos hemos creado los modelos tridimensionales y les hemos aplicado las eventuales modificaciones que necesariamente se extraen de fotografías reales y visitas in situ. Estas fotografías y visitas exigen hacer las modificaciones oportunas para ajustarlos a la verdadera construcción final.

\section{ESENCIA Y SECUENCIA DEL VESTíBULO}

El proyecto para la compañía sAs y el Hotel Royal se inició en el año 1956 cuando se decidió el emplazamiento. Su ubicación central en la ciudad se debió a que se quería concentrar en un mismo complejo una terminal aérea, un hotel y una agencia de viajes, puesto que los tres organismos tenían necesidades complementarias.

El edificio tiene 22 plantas (figs. 4 y 5). En la memoria descriptiva Jacobsen defiende esta solución fundamentalmente por dos motivos. El primero se debe a que el Ayuntamiento exigió que se aprovechara al máximo el potencial que ofrecía el emplazamiento; sin embargo, no hubo otra posibilidad más que completar en su totalidad los $70 \mathrm{~m}$ de altura que 


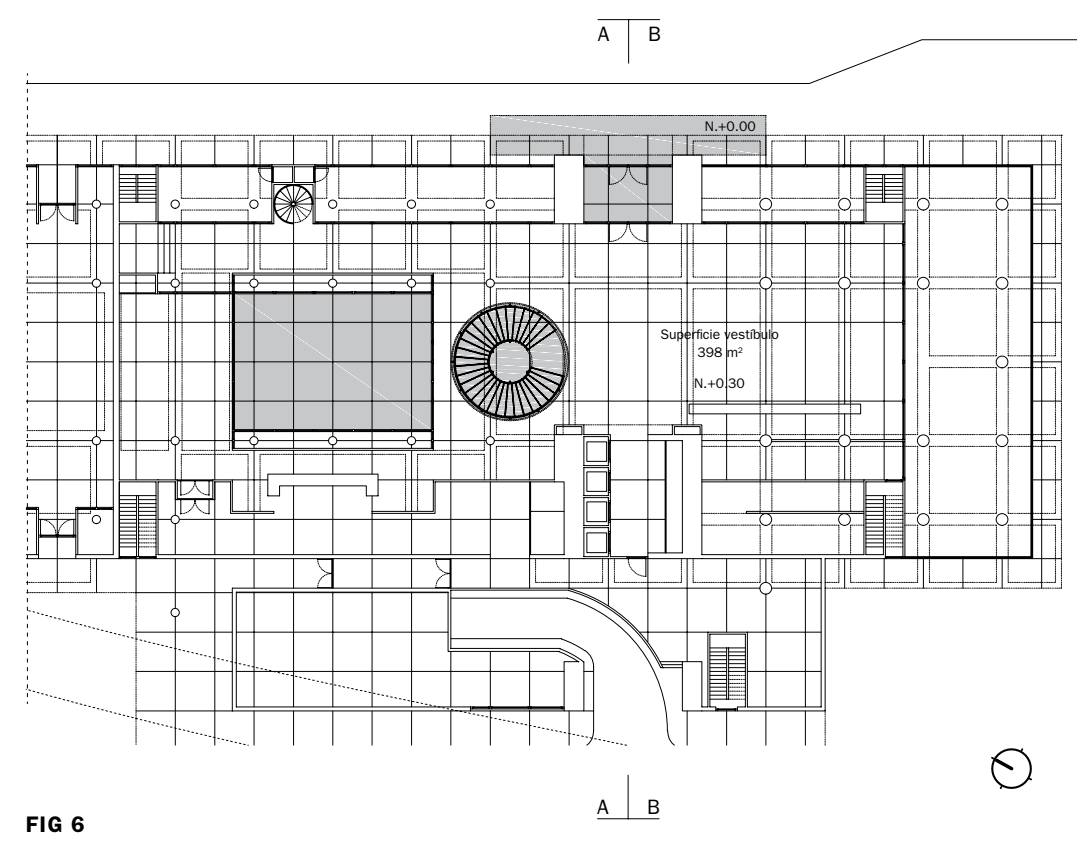

FIG 6

Planta hall principal, nivel $+0,30$

Escala / Scale. 1: 500.

Main hall plan, level $+0,30$.

FIG 7

Corte transversal.

Escala / Scale. 1: 1.000.

Cross Section.

\section{FIG 8}

Planta hall principal, nivel $+5,18$

Escala / Scale. 1: 500.

Main hall plan, level $+5,18$. so más viable para un hotel en un lugar tan ruidoso (2G, 2005 y Solaguren, 1989-2001).

La escalera que analizamos se localiza en el vestíbulo del hotel (fig. 6). Este mismo espacio articula los diferentes programas y usos del edificio. Se accede desde la calle Hammerichsgade potenciando la entrada por la fachada más larga. En la zona de sobresale de la rasante de la fachada y resguarda al permitían las ordenanzas. La segunda razón se debe a que la solución de un edificio en altura era el recurentrada se diseña un elemento, a modo de alero, que visitante delante del acceso inmediato al edificio (fig. 7). Este gesto, que magnifica el entorno urbano invadiendo la zona peatonal, hace que la llegada se produzca de forma paralela a la fachada principal y marca una relación directa con la escalera a modo compositivo. Además comparte rasgos comunes en el diseño estructural, ya que este alero de entrada está suspendido mediante tensores al igual que la escalera helicoidal del interior.
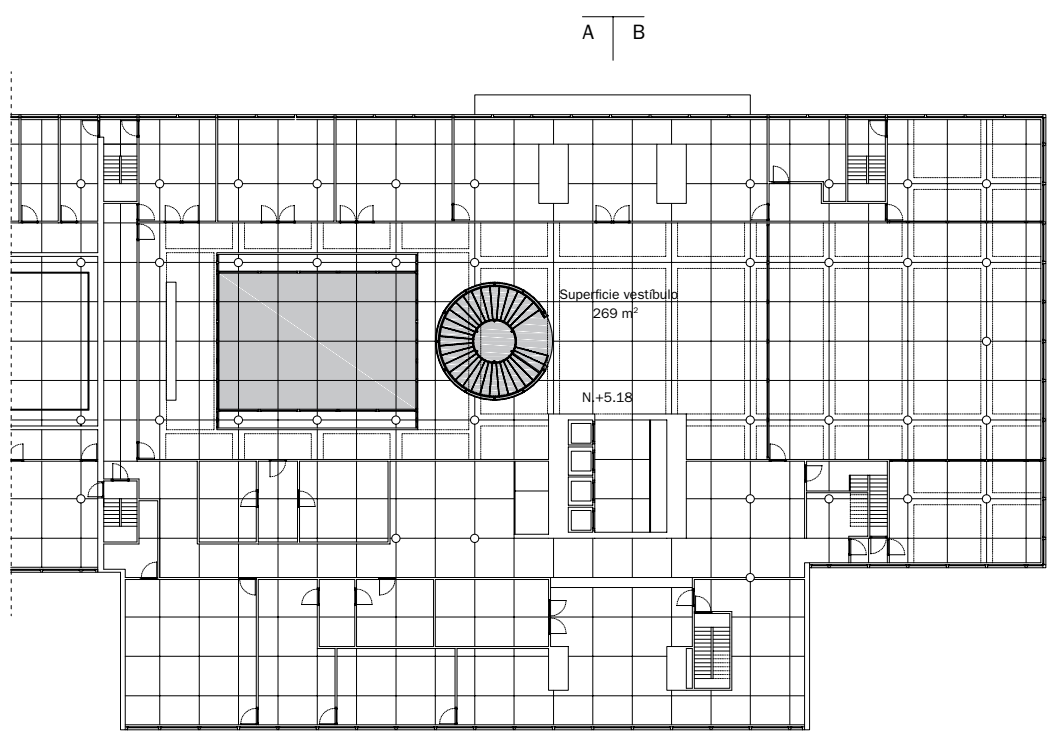

FIG 8

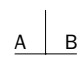

0

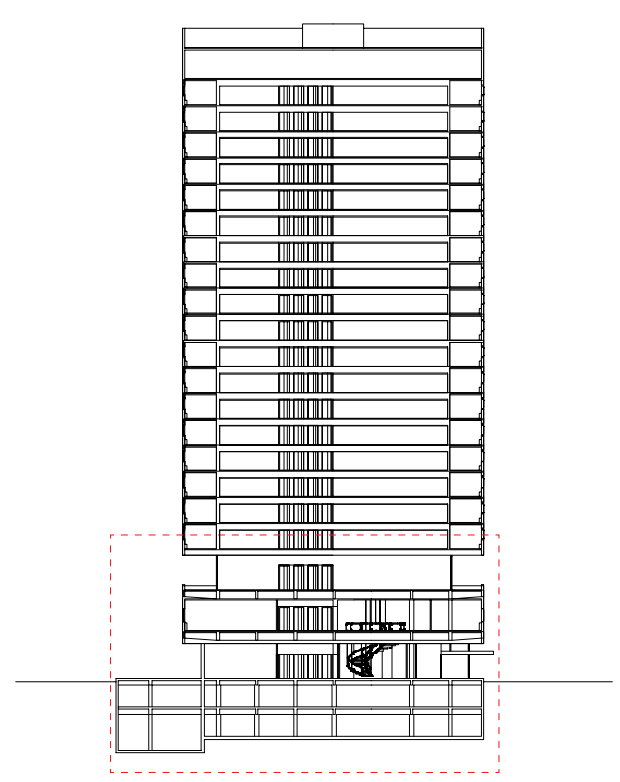

FIG 7

El acceso al vestíbulo del hotel transcurre en medio de dos de los cuatro pilares apantallados que pautan la estructura de la torre. Este espacio, que actúa a modo de cortaviento, queda delimitado horizontalmente por los pilares de 4,5 $\mathrm{m}$ de anchura y verticalmente por la parte inferior de un alero de $2,5 \mathrm{~m}$ de altura que se prolonga hacia el exterior hasta la rasante de la fachada de la torre. Este alero queda empotrado por todo el perímetro en los pilares apantallados y la zona central queda suspendida mediante dos tensores que llegan hasta el techo del vestíbulo. Medio metro por encima de él encontramos un segundo alero que se prolonga $14 \mathrm{~m}$ a lo largo de la fachada principal y sobresale un metro de la rasante. Estos cambios de altura hacen que la secuencia de entrada al vestíbulo desde el exterior suceda de forma gradual. Con esta transición se pretende recuperar la sensación espacial exterior en el interior del vestíbulo.

La geometría del solar y las ordenanzas del Ayuntamiento provocaron que Arne Jacobsen tuviera que diseñar un vestíbulo desdoblado en dos niveles tratando de alcanzar una superficie suficientemente digna para repartir los diferentes programas y usos del edificio. El reto de Jacobsen fue cómo conectar estos dos espacios, separados por 4,88 $\mathrm{m}$ de altura, de una forma natural y elegante por la categoría del edificio. La solución fue diseñar una escalera helicoidal suspendida del techo del nivel superior del vestíbulo, tratando de conectar visualmente un nivel con el otro. Una escalera generosa en dimensiones que protagoniza el vestíbulo de acceso.

El vestíbulo inferior tiene una superficie de 398 $\mathrm{m} 2 \mathrm{y}$ una altura libre de 3,5 $\mathrm{m}$. Este espacio de $10 \mathrm{~m}$ de anchura se puede dividir horizontalmente en tres escenarios: un primer ámbito de acceso y circulación que se enfrenta al núcleo de comunicación de la torre. A la izquierda de este, se encuentra el sector de espera y recepción y, a su derecha, una zona en la que se emplaza un patio cubierto de dos plantas de altura que da luz a la crujía central y que articula a su alrededor un área de restauración; además hace de telón 


\section{FIG 10}

Corte BB / BB Section.

Escala / Scale, 1: 500

Sección transversal realizada por el eje

de acceso al recinto, proyectando la

recepción y abarcando los dos niveles del vestíbulo.

Cross section made through the entrance axis, showing the reception and the two levels of the lobby.

\section{FIG 11}

Vista desde la recepción mirando hacia la escalera.

Fotografía de Aage Strüwing publicada en Edilizia Moderna, Milán, (73): 13, agosto de 1961.

Gentileza de The Aage Strüwing Collection, The Royal Library, Copenhague. View from the reception looking toward the stair

Photography by Aage Strüwing published in Edilizia Moderna, Milan, (73): August 13, 1961. Courtesy of The Aage Strüwing Collection, The Royal Library, Copenhagen.

\section{FIG 12}

Vista desde la escalera mirando hacia la recepción.

Fotografía de Aage Strüwing publicada en SHERIDAN, Michael. Room 606, The SAS House and the Work of Arne Jacobsen. Londres, Phaidon, 2003, p. 117. Gentileza de The Aage Strüwing Collection, The Royal Library, Copenhague.

View from the stair looking toward the reception.

Photography by Aage Strüwing published in sheridan, Michael. Room 606, The SAS House and the Work of Arne Jacobsen. London, Phaidon, 2003, p. 117. Courtesy of The Aage Strüwing Collection, The Royal Library, Copenhagen

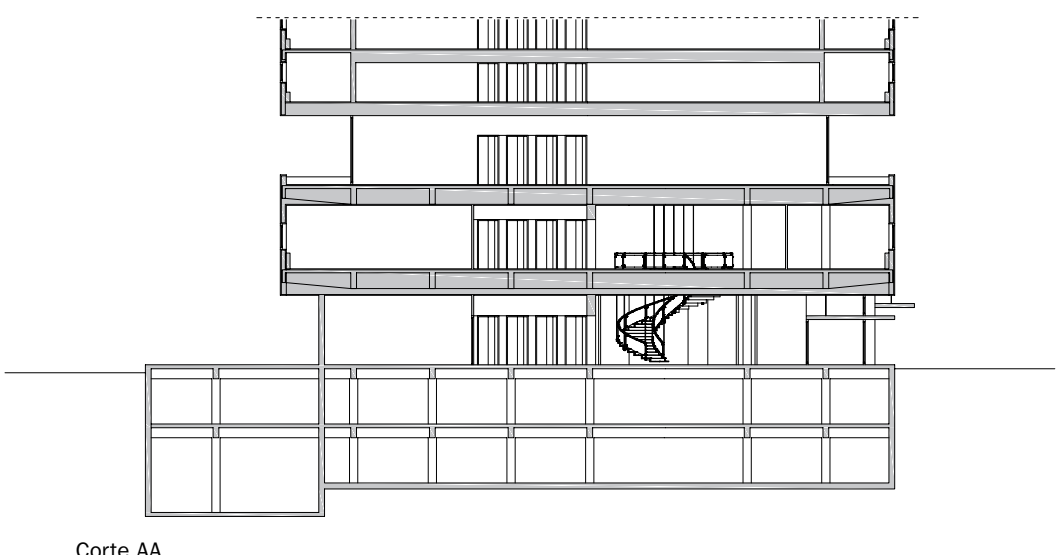

FIG 9

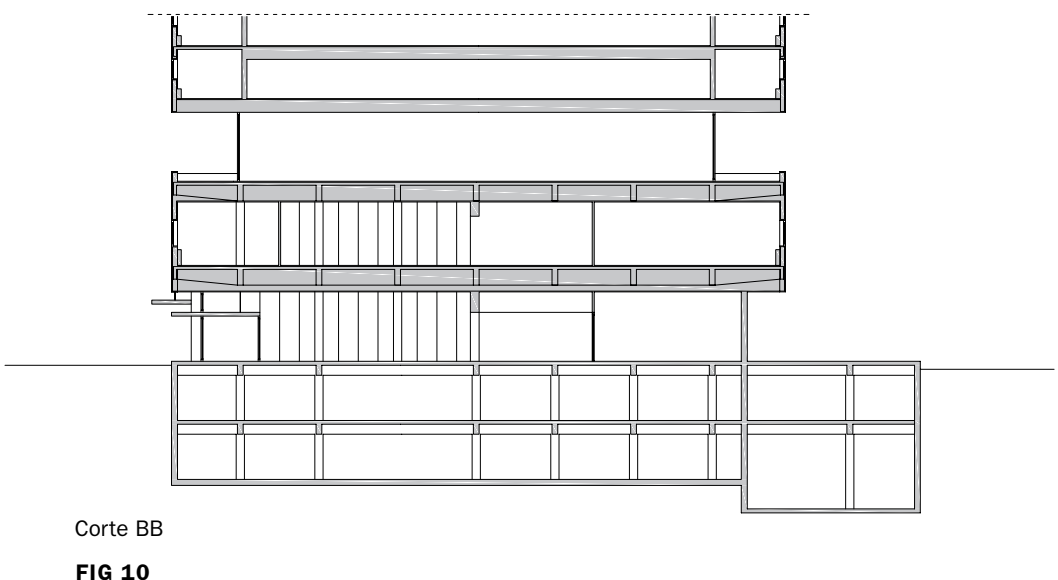

de fondo de la escalera principal. La posición de este elemento de comunicación equidista de todas las zonas del vestíbulo de acceso, ofreciendo una conexión cómoda con el vestíbulo superior. Todo el escenario queda recogido perimetralmente por una zona comercial y técnica de $3 \mathrm{~m}$ de grosor que aísla acústica y visualmente al vestíbulo del exterior.

El vestíbulo superior tiene una superficie de 269 $\mathrm{m} 2 \mathrm{y}$ una altura libre de 3,2 $\mathrm{m}$ (fig. 8). Al igual que el vestíbulo inferior, tiene una anchura de diez metros y el uso de este espacio se puede dividir en dos escenarios; la posición de la escalera en el vestíbulo superior ayuda a separar los programas en este nivel. A la izquierda del anillo perimetral de la escalera encontramos la antesala al restaurante y, a la derecha, el doble espacio del patio cubierto que ilumina y organiza en su perímetro el resto de usos. De la misma manera

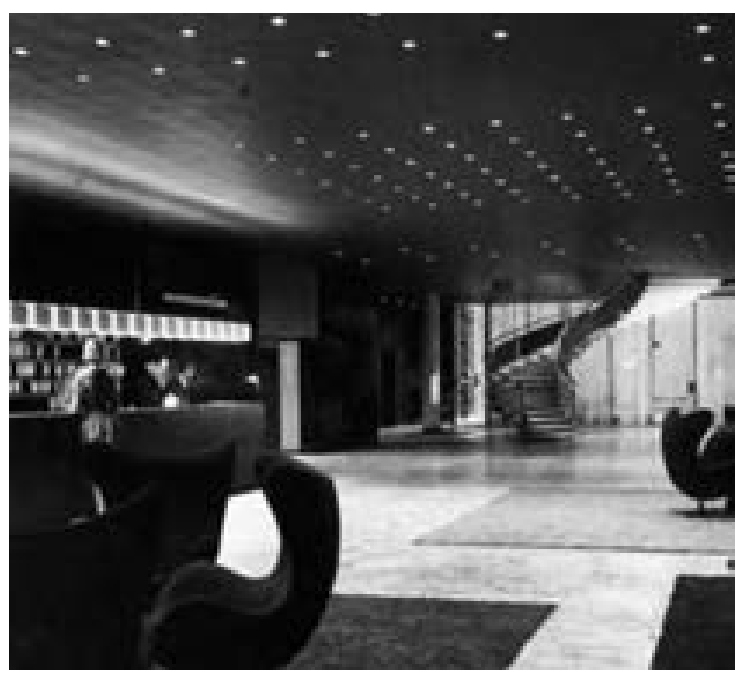

FIG 11 que en el nivel inferior, este vestíbulo queda rodeado perimetralmente por diferentes dependencias aislándolo del exterior. Aparte del patio cubierto, la única relación visual con el nivel inferior son los seis tensores que rodean la parte central de la escalera y que se prolongan casi hasta el suelo del vestíbulo de acceso. Este gesto insinúa que los dos niveles están conectados por uso y funcionamiento a pesar de su posición.

La descripción anterior la hemos obtenido mediante documentos que hemos dibujado expresamente para este trabajo. Hemos elaborado dos secciones verticales hechas por el acceso donde se muestran ambas zonas del vestíbulo: una sección proyectando la escalera y la otra mostrando el extremo opuesto del vestíbulo (figs. 9 y 10). Con este material explicamos gráficamente las relaciones programáticas que tienen los diferentes niveles del vestíbulo con el resto del

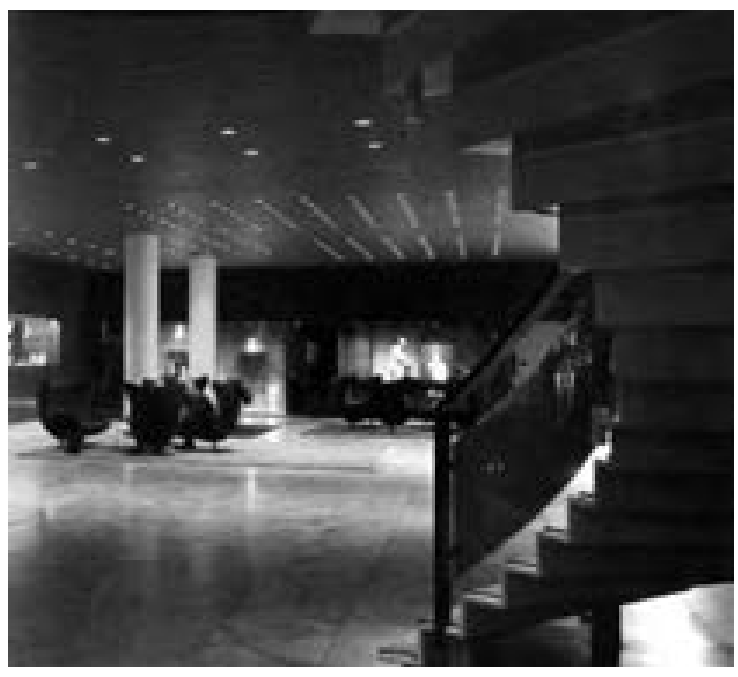

FIG 12 
A la izquierda el acceso principal.

Digital three-dimensional

construction, back view of the hall and stair. View from the stair toward the reception and waiting area. The main entrance to the left.

\section{FIG 14}

Reconstrucción tridimensional digital, vista frontal del hall y escalera.

Digital three-dimensional reconstruction, front view from the hall and stair.

\section{FIG 15}

Vista de la escalera helicoidal desde el vestíbulo de acceso. Fotografía de Aage Strüwing publicada en Edilizia Moderna, Milán, (73): 12, agosto de 1961. Gentileza de The Aage Strüwing Collection, The Royal Library, Copenhague.

View of the spiral stair from the entrance lobby.

Photography by Aage Strüwing published in Edilizia Moderna, Milán, (73): August 12, 1961. Courtesy of The Aage Strüwing Collection, The Royal Library, Copenhagen.

\section{FIG 16}

Detalle constructivo del encuentro entre tensor y anclaje.

Escala / Scale 1:5

Detail of the joint between the cable and anchor.

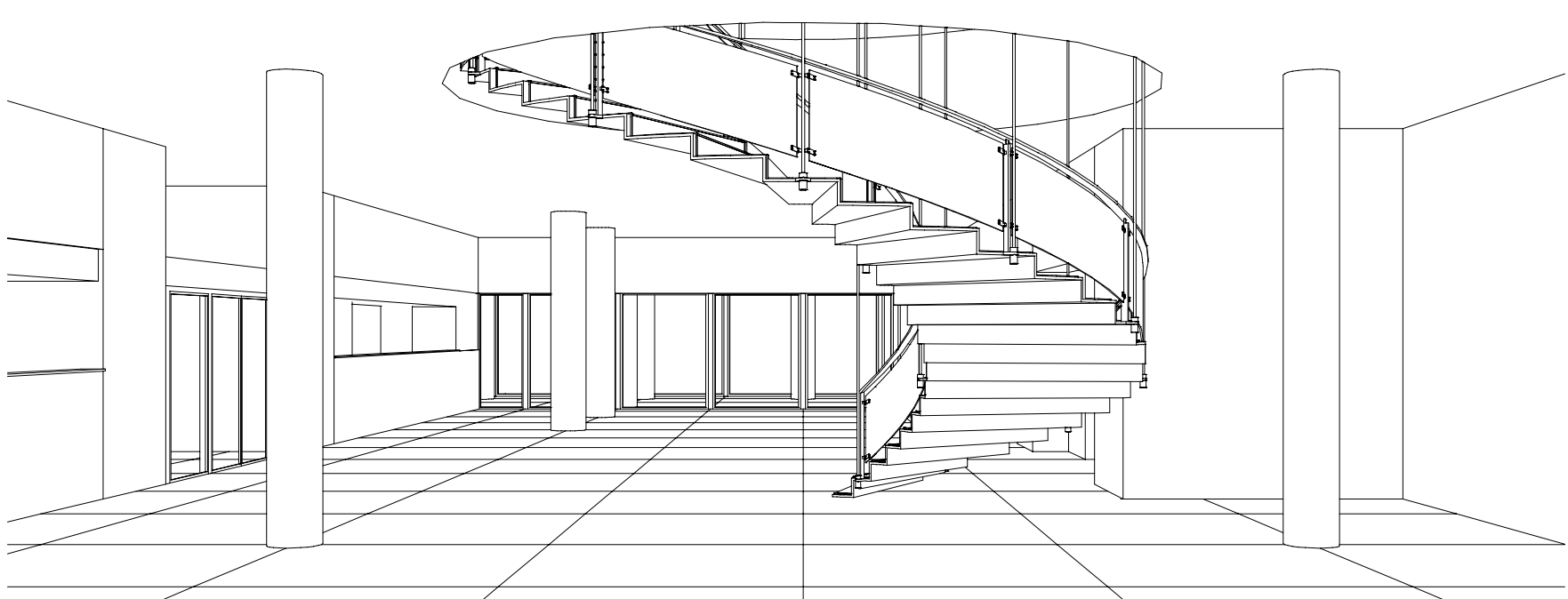

FIG 13

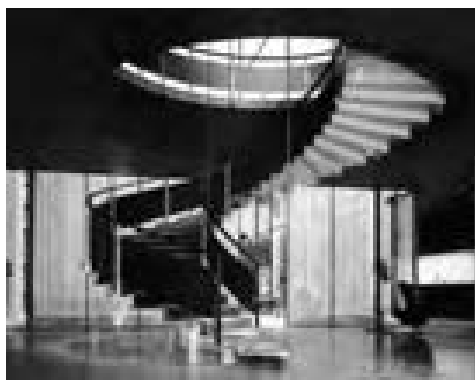

edificio y exponemos la concordancia formal y compositiva que tiene la escalera en relación a este cuando la vemos al entrar o al salir de este espacio.

Paralelamente a las secciones, se incluye la descripción de este espacio y dos fotografías de época del vestíbulo inferior que complementan esa zona (figs. 11 y 12). Al igual que las secciones, una fotografía está hecha desde la recepción mirando la escalera y la segunda desde la escalera hacia la recepción. También hemos hecho una reconstrucción tridimensional del vestíbulo con la finalidad de confirmar que tanto el diseño constructivo de esta escalera como su posición están en relación directa a la composición general del vestíbulo y para confirmar la voluntad de Jacobsen de dotar a la escalera de las mismas cualidades formales que tiene el elemento de acceso y el vestíbulo, generando un ambiente arquitectónico coherente (figs. 13 y 14).

Asimismo podemos observar tres cosas: a nivel visual, el vestíbulo tiene una escasa relación con el exterior a causa de la existencia de un perímetro comercial en el entorno; a nivel compositivo, la escalera está posicionada con el ángulo idóneo para encarar el primer peldaño en dirección a la puerta de acceso; y finalmente, hay que considerar a nivel estructural que la escalera, a pesar de estar suspendida mediante 16 tensores, en el segundo peldaño contiene dos soportes sutilmente disimulados que se apoyan en el suelo y que anulan las posibles vibraciones al subir o bajar por este elemento de comunicación (fig. 15).

\section{FORMA Y MATERIAL}

La escalera del vestíbulo del Hotel Royal sAs es de tipología helicoidal, formada por 32 peldaños, salvando una diferencia de cota de 4,88 $\mathrm{m}$. Este desnivel se completa dejando libre una octava parte del recorrido de una vuelta (AA.VV., 1983).

El tramo helicoidal tiene una anchura libre de paso de 1,8 metros, equivalente a la anchura de cada peldaño, y el ojo central tiene un diámetro de 2,4 metros. El conjunto de escalones están formados mediante una lámina metálica de color blanco que gira ajustándose a la curvatura, un acabado de moqueta antideslizante y unos perfiles biselados en las aristas horizontales para rematar dicho acabado. La lámina metálica continua está estructuralmente suspendida mediante 16 tensores que se distribuyen circularmente a lo largo del perímetro interior y exterior de la escalera (fig. 16).

Encontramos dos tipos de barandillas: una primera que va siguiendo el recorrido helicoidal en su perímetro exterior y en el ojo de la escalera, y una segunda que tiene un recorrido horizontal y que va volteando el agujero circular que produce la escalera con el suelo del vestíbulo superior. A pesar de sus diferencias 


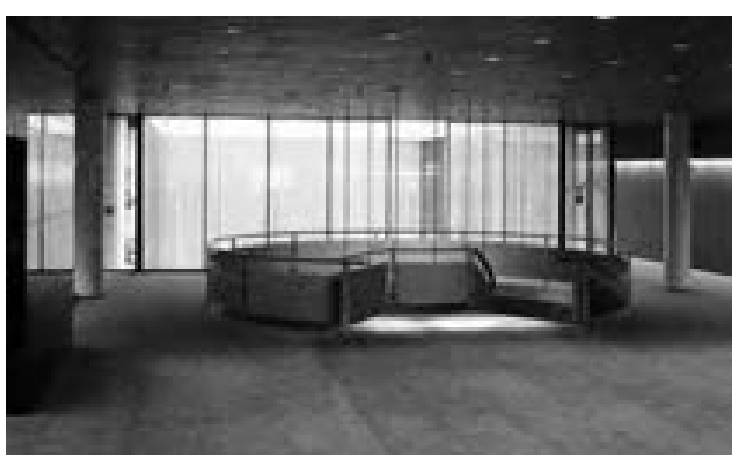

FIG 17
FIG 17

Vista de la escalera helicoidal desde el vestíbulo superior.

Fotografía de Aage Strüwing publicada en Techniques et architecture, París, 21 (2): 140, 1961.

Gentileza de The Aage Strüwing

Collection, The Royal Library,

Copenhague.

View from the spiral stair toward the upper lobby.

Photography by Aage Strüwing published in Techniques et architecture, Paris, 21 (2): 140, 1961.

Courtesy of The Aage Strüwing Collection, The Royal Library, Copenhagen.

\section{FIGS 18A- 18E}

Reconstrucción bidimensional de la escalera helicoidal.

Two-dimensional reconstruction of the spiral staircase.

A Planta superior / Upper plan.

B Planta intermedia / Intermediate plan.

C Corte CC / CC Section.

D Corte DD / DD Section.

E Elevación / Elevation.

Escala / Scale 1: 20
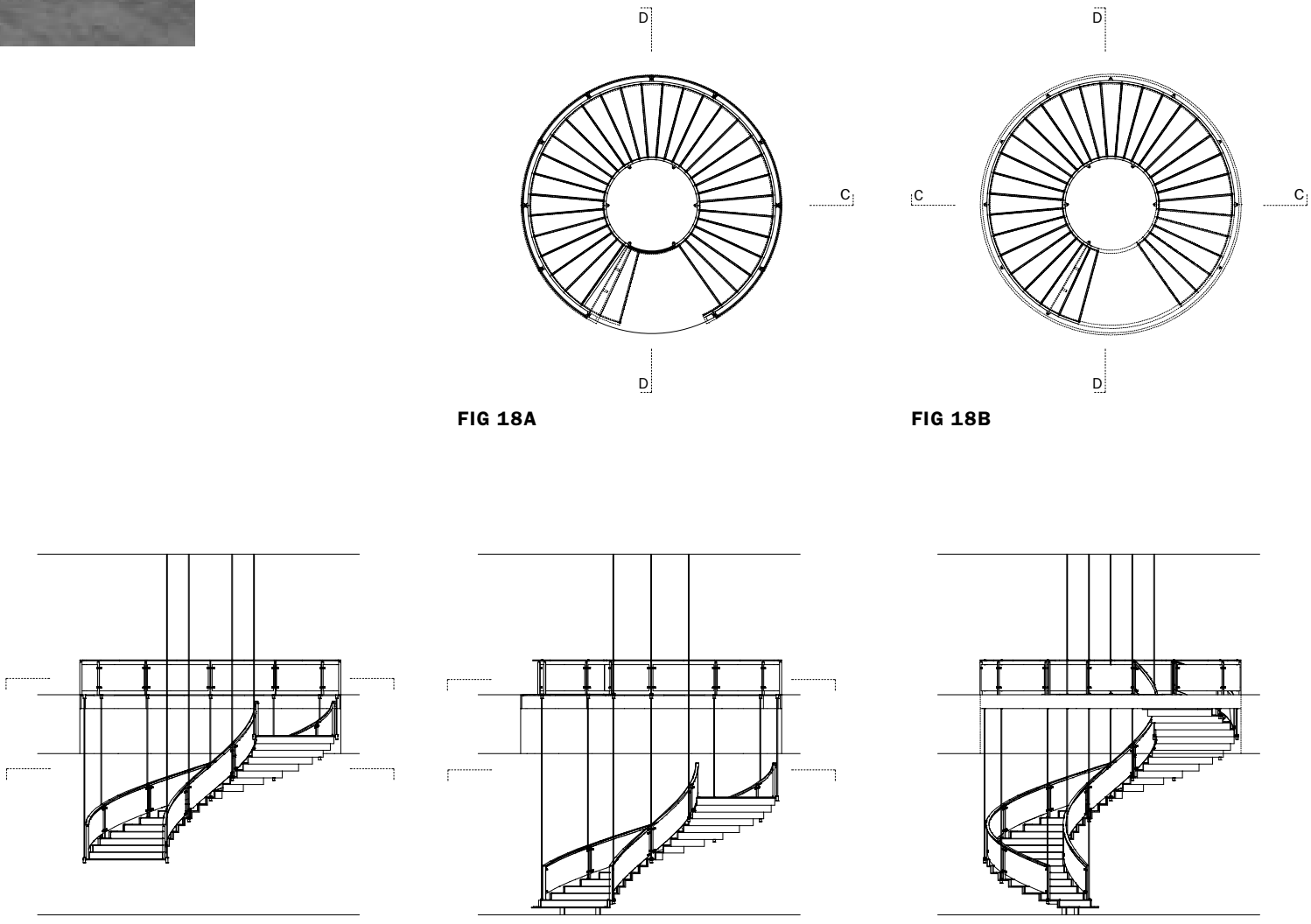

FIG 18D

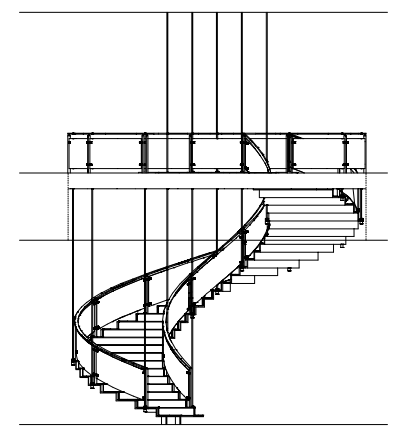

FIG 18E
FIG 18C

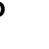

geométricas, a nivel formal las podemos describir de la siguiente manera: barandilla de $90 \mathrm{~cm}$ de altura formada por un travesaño superior metálico, de sección rectangular y color gris oscuro, y unos montantes verticales de color blanco que se van repitiendo cada $60^{\circ}$. El espacio que delimita los montantes y los travesaños se completa mediante un vidrio oscurecido que se encuentra anclado por cuatro piezas engastadas a los montantes verticales y que resisten las torsiones. En la zona de la escalera el vidrio sigue una curvatura helicoidal, mientras en la zona superior se ajusta a la curvatura circular (figs. 17 a 20).

En el perímetro exterior de la escalera encontramos un total de diez tensores, uno cada $30^{\circ}$. Estos tienen diferentes longitudes y van desde la chapa plegada hasta el perímetro interior del agujero del nivel superior. En el ojo de la escalera encontramos seis tensores cada $60^{\circ}$ que están alineados en planta con los tensores del exterior y que van desde la chapa plegada hasta el techo del vestíbulo superior.

Arne Jacobsen quiso remarcar la funcionalidad de cada uno de los elementos de la escalera dotándolos de un color; por este motivo, los tensores están pintados de color rojo, la chapa plegada y los montantes verticales de color blanco y el pasamanos de color gris oscuro. Su condición de arquitecto y de ingeniero industrial hizo que empleara y definiera como carac- terización tecnológica de su diseño y de su determinación formal, el uso de componentes industriales. Jacobsen se identificó con la influencia de estas nuevas técnicas en la forma arquitectónica, favoreciendo los presupuestos y potenciando la cultura tecnológica aplicada a elementos de arquitectura (Habermann, 2003 y Pracht, 1991).

\section{ESTRUCTURA}

Para entender bien el comportamiento estructural de la escalera, primero se ha de entender el comportamiento estructural de la porción de edificio donde está suspendida. El vestíbulo se define estructuralmente mediante una crujía central de $8 \mathrm{~m}$ de ancho delimitada por dos hileras de pilares separados cada $4 \mathrm{~m}$. La escalera se sitúa en medio de esta crujía central y en un extremo del vestíbulo. Su conexión con el nivel superior, losa superior de hormigón armado con vigas de gran canto, genera un agujero que relaciona los dos espacios y, estructuralmente hablando, se remata con un zuncho circular, a modo de pletina metálica, que se conecta de forma monolítica con el resto de la losa.

La escalera está generada por una pletina soldada de acero de $2 \mathrm{~cm}$ de grosor y con 32 pliegues. En esta lámina se descarga el peso de todos los acabados $\mathrm{y}$ partes de las barandillas. Todo este sumatorio de 
Detalle del contacto de la escalera con el suelo.

Fotografía de Aage Strüwing publicada en Edilizia Moderna, Milán, (73): 13, agosto de 1961.

Gentileza de The Aage Strüwing

Collection, The Royal Library,

Copenhague.

Detail of the connection of the stair to the ground.

Photography of the Aage Strüwing

published in Edilizia Moderna, Milan, (73): August 13, 1961.

Courtesy of The Aage Strüwing Collection,

The Royal Library, Copenhagen.

\section{FIG 20}

Detalle de la llegada de la escalera al nivel superior.

Fotografía de Aage Strüwing publicada en SHERIDAN, Michael. Room 606, The SAS House and the Work of Arne Jacobsen.

Londres, Phaidon, 2003, p. 148

Gentileza de The Aage Strüwing Collection, The Royal Library,

Copenhague.

Detail of the arrival of the stair to the upper level.

Photography by Aage Strüwing published in sheridan, Michael. Room 606, The SAS House and the Work of Arne Jacobsen. London, Phaidon, 2003, p. 148.

Courtesy of The Aage Strüwing Collection, The Royal Library, Copenhagen.

\section{FIG 21}

Axonométrica / Axonometric.

s. /E. / Not to scale.

A. Barra de acero de $24 \mathrm{~mm}$ de sección, pintada de rojo. / $24 \mathrm{~mm}$ width steel bar. painted red.

B. Vidrio endurecido de $10 \mathrm{~mm}$ espesor. / Hardened glass $10 \mathrm{~mm}$ wide.

C. Acabado de moqueta antideslizante y absorbente acústico de $1 \mathrm{~mm}$ de espesor, de color azul. / Finished with anti-slip and acoustic absorbing carpet $1 \mathrm{~mm}$ thick, blue color.

D. Perfil metálico de sección en forma de L como guía de la moqueta. / L-section metal profile as finish guide.

E. Perfil metálico biselado para cantos de escalón. / Beveled steel section for step edges.

F. Anclajes de acero inoxidable

resistentes a torsión, atornillados. / Stainless steel anchors resistant to torque, screwed.

G. Pletina soldada de acero de $20 \mathrm{~mm}$ de espesor, lacada de color blanco. / $20 \mathrm{~mm}$ wide steel strip, white lacque

$\mathrm{H}$. Sujeción barras enroscadas de acero.

Steel threaded bars fastening.

I. Montantes verticales de acero

inoxidable de $50 \times 20$ mm de sección, lacados de color blanco. / Stainless steel uprights $50 \times 20 \mathrm{~mm}$, white lacquer. J. Barandilla rectangular de acero inoxidable de 70 × $20 \mathrm{~mm}$ de sección, lacada de color gris oscura. / Stainless steel rectangular handrail $70 \times 20 \mathrm{~mm}$ dark gray lacquer.

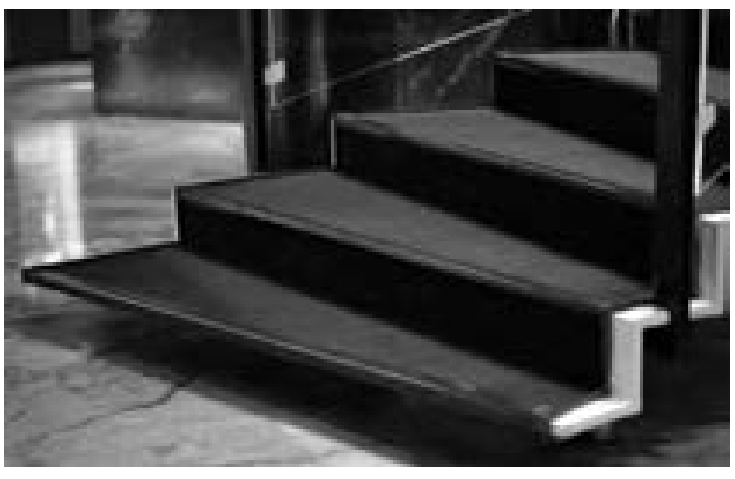

FIG 19

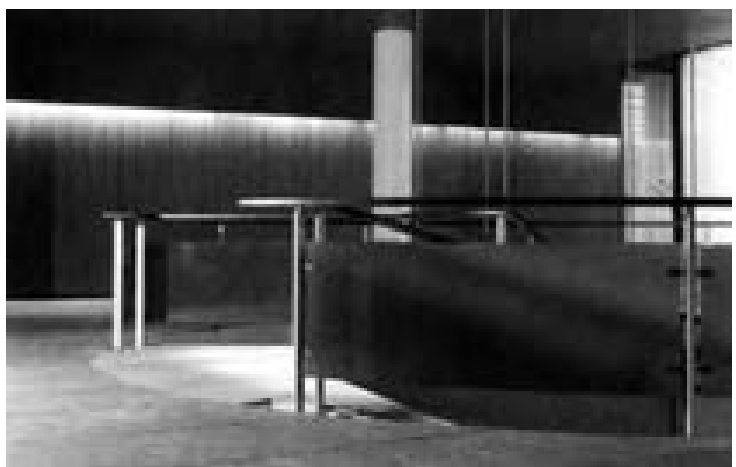

FIG 20

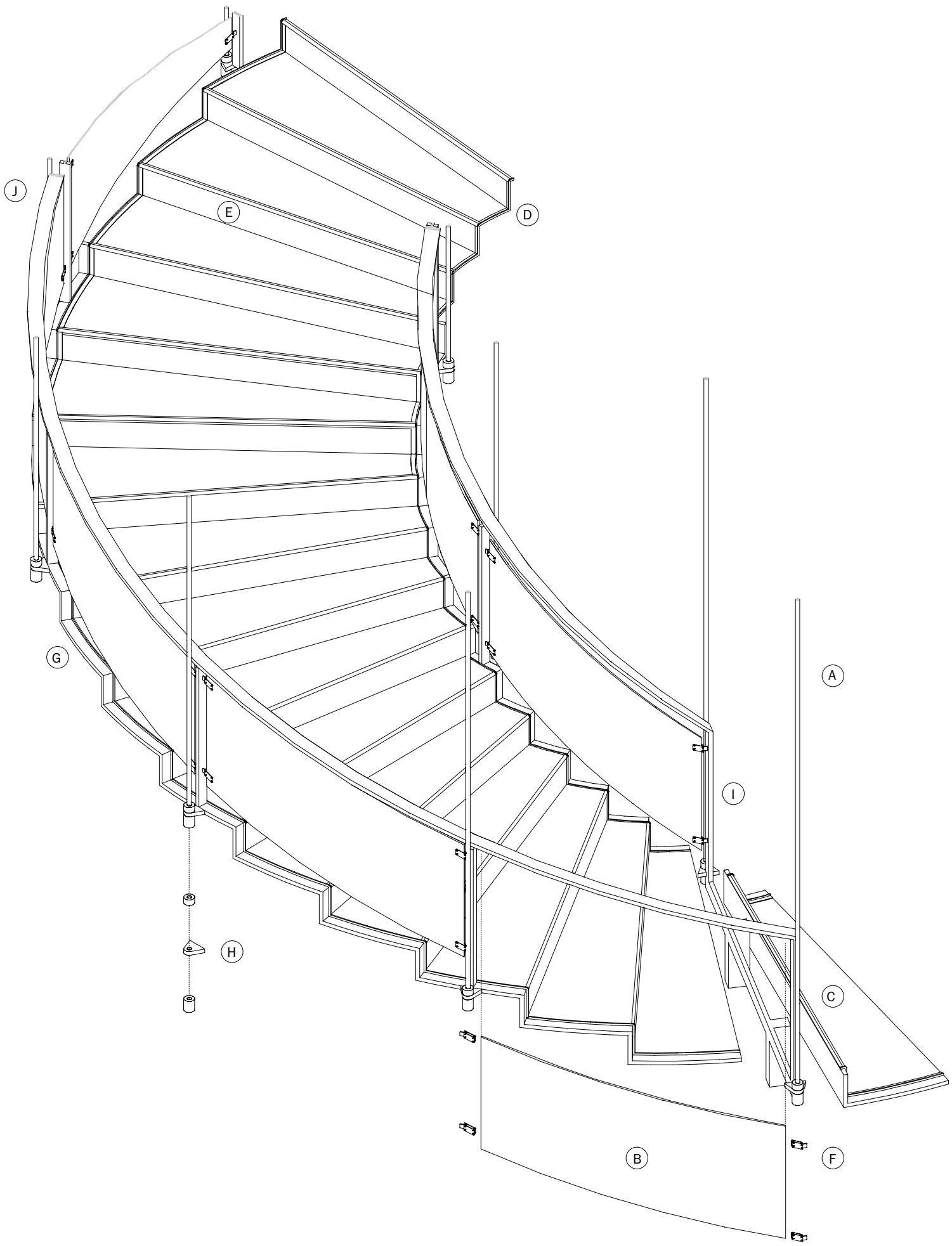

FIG 21 
cargas se transmite proporcionalmente a los 16 tensores de acero de $24 \mathrm{~mm}$ de diámetro que distribuyen las tensiones a los forjados del edificio. Como hemos dicho, diez tensores cada $30^{\circ}$ en su perímetro circular exterior y seis tensores cada $60^{\circ}$ en torno al ojo de la escalera. Cada tensor tiene una longitud diferente debido a que los tensores exteriores van de la lámina de acero hasta el zuncho circular del suelo del vestíbulo superior y los tensores interiores van de la lámina de acero hasta el techo del vestíbulo superior. La deformación vertical de cada una de estas barras, que terminan en rosca, se puede regular a través de cada uno de los conectores (fig. 21).

Se debe considerar que en la parte inferior del segundo peldaño encontramos dos soportes que contactan con el suelo del vestíbulo de acceso para anular las vibraciones al subir o bajar por este elemento de comunicación. Puntualizamos que en 1961 Jacobsen diseñó una escalera lineal totalmente suspendida por ocho tensores en el vestíbulo del Banco Nacional de Dinamarca y en ese caso no existe ningún contacto con el suelo. Por lo tanto, se observa que Arne Jacobsen ensaya métodos técnicos en cada uno de sus diseños y los va perfeccionando a lo largo de su trayectoria profesional (AA.VV., 1961; Faber, 1964 y Pracht, 1991).

\section{DISCUSIÓN Y CONCLUSIONES}

En el presente trabajo reflejamos con fidelidad la escasa documentación original obtenida. A partir de la misma, elaboramos un modelo tridimensional y presentamos documentación amplia con la que se pueden destacar los diferentes elementos de estudio.

Con este análisis, cualquier arquitecto interesado en la escalera helicoidal de Arne Jacobsen puede obtener datos que no se encontraban, hasta el presente artículo, a disposición pública de forma clara.

Además concluimos que es la primera escalera suspendida helicoidal de la historia que se colgó íntegramente mediante 16 tensores y utilizando una lámina de acero plegada como elemento unificador. Hasta ese momento, el diseño estructural más común para colgar estos elementos consistía en suspender cada uno de los peldaños que configuraban el sistema o en usar zancas perimetrales que desempeñaban la misma función. Posterior a la escalera helicoidal del Hotel Royal sAs encontramos varias escaleras helicoidales que tienen rasgos técnicos y constructivos muy parecidos, confirmando así la repercusión técnica que tuvo el diseño de la escalera de Jacobsen.
Del mismo modo, en el proyecto donde se localiza esta escalera, existe un elemento de acceso al edificio que también está suspendido. Con esto, remarcamos que el diseño de la escalera repercute a nivel compositivo en el diseño del vestíbulo y que la decisión de colgarla se hace en consonancia con otro elemento del mismo espacio, destacando así la dualidad como motivo y orden de proyecto.

Finalmente, después de una detallada búsqueda bibliográfica, podemos afirmar que el presente artículo es el primer documento que muestra públicamente el análisis funcional, formal y estructural de la escalera helicoidal del Hotel Royal sAs. Así, con este trabajo, ponemos al alcance de arquitectos, o profesionales de otras áreas, una nueva fuente de ideas sobre la técnica y filosofía de Arne Jacobsen. ARQ

\section{REFERENCIAS BIBLIOGRÁFICAS}

2G. Arne Jacobsen: Edificios Públicos, Barcelona, (4), 1997. AA.VV. Techniques et architecture, París, 21 (2), 1961.

AA.VV. Daidalos. Berlin Architectural Journal, Berlín, (15), 1983. FABER, Tobias. Arne Jacobsen. Stuttgart, Verlag Gerd Hatje, 1964.

HABERMANN, Karl. Staircases. Design and Construction. Berlín, Birkhauser, 2003.

HOFFMANN, Kurt y GRIESE, Helga. Escaleras de acero. Barcelona, Blume, 1970.

JIRICNA, Eva. "Stairway in a Fashion Shop in London". Detail (2): 156-158, abril-mayo de 1990.

JIRICNA, Eva. Staircases. Singapur, Laurence King Editorial, 2001.

MC CARTER, Robert. Fallingwater, Frank Lloyd Wright. Londres, Phaidon, 1994

PELKONEN, Eeva-Liisa y ALBRECHT, Donald (eds.). Eero Saarinen, Shaping The Future. New Haven, Yale University Press, 2011. PRACHT, Klaus. Escaleras interiores y exteriores. Barcelona, Gustavo Gili, 1991.

SÁNCHEZ, Ginés. "Historia de una escalera, Eva Jiricna para Joseph". Arquitectura Viva (10): 62-63, enero-febrero de 1990. SLESSOR, Catherine. Escaleras contemporáneas. Barcelona, Gustavo Gili, 2001.

SOLAGUREN, Félix. Arne Jacobsen: Obras y Proyectos. Barcelona, Gustavo Gili, 1989.

SOLAGUREN, Félix. Arne Jacobsen. Barcelona, Santa \& Cole Ediciones, 1991.

SOLAGUREN, Félix. Arne Jacobsen: Aproximación a La Obra Completa 1950-1971. Barcelona, Fundación Caja de Arquitectos, 2001.

ZABALBEASCOA, Anatxu. Las casas del siglo. Barcelona, Gustavo Gili, 1998.

\section{BibLIOGRAFÍA}

DE LONG, David y FORD PEATROSS, C. Eero Saarinen. Nueva York, W. W. Norton Editorial, 2008.

EDILIZIA MODERNA, Milán, (73), agosto de 1961.

SHERIDAN, Michael. Room 606, The SAS House and the Work of Arne Jacobsen. Londres, Phaidon, 2003.
ALBERT SAMPER | Arquitecto, Universidad Politècnica de Catalunya (UPC), 2008; Máster en Teoría y Práctica del Proyecto Arquitectónico, UPC, 2011 y Doctor, Ph.D., Universidad Rovira i Virgili, 2014. La línea central de su trabajo es el análisis geométrico de elementos arquitectónicos. Actualmente es profesor de Geometría en el Área de Expresión Gráfica Arquitectónica de la Universidad Rovira i Virgili. Sus principales campos de interés son la geometría fractal y la aplicación de la geometría a la arquitectura.
BLAS HERRERA | Matemático y Geómetra, Universidad de Barcelona, 1991; Máster en Matemáticas, Universidad Autónoma de Barcelona (UAB), 1993 y Doctor en ciencias, Sc.D., UAB, 1994. La línea central de su trabajo es la geometría y su computación aplicada. Actualmente es profesor de Matemática Aplicada de la Escuela Técnica Superior de Arquitectura de la Universidad Rovira i Virgili. Sus principales campos de interés son la geometría clásica y diferencial aplicada a la arquitectura, la mecánica de fluidos y la ingeniería. 
ALBERT SAMPER | PROFESSOR, PRE-DEPARTMENTAL UNIT OF ARCHITECTURE, UNIVERSITAT ROVIRA I VIRGILI, TARRAGONA, SPAIN.

BLAS HERRERA | PROFESSOR, DEPARTMENT OF INFORMATION ENGINEERING AND MATHEMATICS, UNIVERSITAT ROVIRA I VIRGILI, TARRAGONA, SPAIN.

\section{THE SPIRAL STAIRCASE OF THE HOTEL} ROYAL SAS, ARNE JACOBSEN

The first hanging stairs appeared at the beginnings of the last century. Leading architects like Rudolf Schindler or Richard Neutra began to design suspended linear stairs in single-family dwellings: the first attempts consisted in suspending each step on one end and cantilever the other side. Hanging stairs began to emerge in 1935 when the architect Frank Lloyd Wright designed a linear hanging stair in Falling Water (Mc Carter, 1994 and Zabalbeascoa, 1998). The constructive system of this stair was partially similar to Schindler and Neutra's system in that it consisted of hanging each step in such a way that one rod traverses two steps so as to counteract vibrations and torque.

After the Second World War-and with the influence of North American technological and constructive systems disseminated in publications such as Progressive Architecture-the steel construction system was improved and permitted the application of new structural formulas in stair design. Arne Jacobsen was one of the first architects to adopt the possibilities that steel permitted: an example of this attitude is the integrally suspended design of the spiral staircase in the vestibule of the Hotel Royal sAs in Copenhagen, whose project was developed between 1956 and 1961. Another spiral staircase with very similar technical and constructive features to the one designed by Jacobsen (and a contemporary) was that which architect Eero Saarinen built in the lobby of the General Motors Technical Center $^{1}$ (fig. 1) (Long and Peatross, 2008; Pelkonen and Albrecht, 2011). A third example that embodied these new developments was the spiral staircase in the Canadian Pavilion² (fig. 2) (Hoffmann and Griese, 1970) one of the many displays of new building concepts designed for steel presented at the Universal Exposition of Brussels in 1958.

Both the designs executed by Arne Jacobsen and those presented in the Universal Exhibition confirm the technical impact and will for innovation in the design of hanging stairs. Another clear example is the spiral staircase designed by the architects Friedrich Wilhelm, Günter Pfenning and Ernst Sieverts for the lobby of the Administration Building of the city of Brunswick in 1959. Nowadays, there are many examples of hanging stairs that due to progress in construction have been able to hide, as much as possible, any kind of building detail with the goal of making this element of communication levitate (Slessor, 2001 y Sánchez, 1990). A representative example of this kind of design is the stair in the Joseph store (fig. 3) by the architect, Eva Jiricna, built in 1989 in London (Jiricna, 1990 and 2001).

For these reasons, the staircase at the Hotel Royal sAs deserves a personalized study. We intend to define it through the spaces and elements of the building that are designed around it, attempting to discover the intention of each of the gestures of this staircase until we discover all the layers that make up the construction detail.

Our main objective is to reveal each detail and material. We intend to stage a two-dimensional and three-dimensional reconstruction to clarify historical graphic documentation that does not clearly describe this element. And with this information, we will explain the formal reasoning behind the design of the staircase suspended in the main lobby of the Hotel Royal sas.

\section{MATERIAL Y METHOD}

In undertaking the investigation, the main source of information has been periodicals from that time, such as Edilizia Moderna and Techniques et Architecture. With the general information that we have found in these registries we have been able to generate the graphic documents that relate the stair with the architecture and design of the building. A great many monographs exist on Arne Jacobsen. However, few are of the level necessary to be considered as a point of reference in the current investigation. The one that offers the most information and in which more technically details can be seen is the monograph Classics of Design of Arne Jacobsen (Solaguren, 1991).

As for the wealth of information offered by Internet sources, clarification must be made to test and then treated to improve the focus on the concept. Specialized search engines such as Google Academic, the library of the Colegio Oficial de Arquitectos de Cataluña, the Catalogue Universitat Politécnica de Catalunya or the datebase of the Royal Institute British Architects, have been the main tools for inquiry because of the rigor in the information and their proximity.

In order to carry out the present work, which requires new perspectives, we have re-elaborated all of the original documents with the necessary rigor to create a new one altogether. We have created new three-dimensional models. The document is provided with the necessary qualities to convey the study. The reconstruction process was made from the observation of graphic documents, be they photographs or technical plans. Subsequently, a record of the information has been made using programs such as AutoCad, for making the two-dimensional reconstruction, and Google Sketchup, for making the threedimensional reconstruction.

The process begins by compiling the best information possible for creating an exhaustive description that would allowing us to observe the totality of the project and begin analysis. We continue with a subsequent quality digitalization of all the documents, plans or photography. The new drawings are faithful to the original, but line weights were adjusted, using their values and the appropriate sections to highlight the desired circumstances. Basing the work on these new drawings, we have created three-dimensional models and have applied the modifications observed in the real photographs and on site visits. These photographs and visits require us to make the necessary modifications to adjust to the real final construction.

\section{ESSENCE AND LOBBY SEQUENCE}

The project for the SAS Company and the Hotel Royal began in 1956 when the site was decided upon. Its central location in the city was to the desire to concentrate an airport terminal, hotel and travel agency in the same complex, as the three organisms had complementary needs.

The building has 22 floors (figs. 4 and 5). In the descriptive summary Jacobsen defends this solution fundamentally for two reasons. The first is that the city demanded that the site should be exploited to its maximum capacity; however, there was no alternative but to complete the total 70 meters of height permitted by code. The second reason was due to the solution of a high-rise building was the most viable option for a hotel in such a noisy location $(2 G, 2005$ and Solaguren, 1989-2001).

The staircase that we analyzed was located in the hotel lobby (fig. 6). This space articulates the different programs and uses of the building. It is accessed from Hammerichsgade Street, enhancing the entrance on the longest facade. In the entrance area and element was designed like an eave that comes out of the facade and protects the visitor's immediate access of the building (fig. 7). This gesture, that magnifies the urban environment invading the pedestrian zone, means the arrival occurs in parallel to the main facade and compositionally creates a direct relationship with the stair. It also shares common features with its structural design, as it is also a cable-suspended element.

Access to the hotel lobby occurs between two of the four screen-like pillars that make up the tower structure. This space that acts as a windbreak is bounded horizontally by these pillars -4.5 meters wide- and vertically by the lower part of an eave 2.5 meters high that extends outward to the facade of the tower. This eave is set into the pillars along the whole perimeter; two cables that run up to the ceiling of the lobby suspend its central area. Half a meter above we find a second eave that extends 14 meters along the main facade and jets out a meter from it. These height changes create a gradual entrance sequence. This transition seeks to recover the spatial sensation of the exterior within the lobby.

Solar geometry and city building code provoked Arne Jacobsen to design a splitlevel lobby in order to accommodate the different building programs and uses. Jacobsen's challenge was to connect these two spaces, separated in height by 4.88 meters, in a natural and elegant way. The solution was to design a spiral stair suspended from the ceiling of the upper level of the lobby that attempts to connect one level with the other visually. The generously large stair becomes the focus of the entrance lobby.

The interior lobby has a surface of $398 \mathrm{~m}^{2}$ and a height of 3.5 meters. This 10 meters wide space can be divided horizontally in three areas: a first entrance area and circulation that faces the communication nucleus of the tower. To the left of this, a waiting area and reception are found, and to the right a zone a covered patio of two tall plants that gives light to the central bay articulating an area of restoration around it and acting as a backdrop to the main staircase. The position of this communication element is equidistant from all zones in the entrance lobby and offers a comfortable connection with the upper lobby. The whole scene is contained along the perimeter by a commercial and technical area 3 meters thick that acoustically and visually isolates the lobby from the exterior.

The upper lobby has an area of 269 $\mathrm{m}^{2}$ and a height of 3.2 meters (fig. 8). Like in the lower lobby, it has a width of 10 meters and the use of this space can be divided into two scenes; the position of the stair in the upper lobby helps to separate the program on this level. To the left of the perimeter ring we find the antechamber to the restaurant and to the right the double space of the covered patio that illuminates and organizes the rest of the uses in the perimeter. In the same way as below, different programs surround the lobby, isolating it from the exterior. Apart from the covered patio, the only visual relationship with the lower level is that of the six cables at the central part of the stair; they extend almost to the floor of the entrance lobby. This gesture insinuates that the two 
levels are connected by use and function despite their position.

The above description we have obtained through documents that we have drawn expressly for this work. We have elaborated two vertical sections taken through the access that show both areas of the lobby: a section showing the stair and another showing the extreme opposite of the lobby (figs. 9 and 10). With this material we graphically explain the programmatic relationships that the levels of the lobby establish with the rest of the building and we expose the formal and compositional consistency that the stair has in relation to the lobby, when we see it upon entering or leaving this space.

Parallel to the sections, a description of this space is included as well as two photographs of the lower lobby aiming to show that zone (figs. 11 and 12). Like the sections, a photograph is taken from the reception looking at the stair and the second from the stair to the reception. We have also made a three-dimensional reconstruction of the lobby with the aim of confirming the construction design of this stair and its position in direct relation to the general composition of the lobby and to confirm Jacobsen's desire to give the stair the same formal qualities as the access element and the lobby, generating a coherent architectonic environment (figs. 13 and 14).

We can also observe three things: visually wise, the lobby has a poor relationship with the outside due to the existence of a commercial perimeter; as a composition, the stair is positioned with the ideal angle to place the first step in the direction of the access door; finally, we must consider on a structural level that the stair, despite being suspended by 16 cables, has two supports that rest on the floor subtly concealed in the second step. These supports nullify the possible vibrations from going up or down (fig. 15).

\section{FORM AND MATERIAL}

The lobby stair of the Hotel Royal sas is of a spiral typology, formed by 32 steps, and 4.88 meters high. This height is completed, leaving an eighth of a turn free (AA.VV., 1983).

The spiral section has a width 1.8 $\mathrm{m}$, equivalent to the width of each step, and the central eye has a diameter of $2.4 \mathrm{~m}$. The set of steps are formed with a white metal sheet the turns to adjust to the curve, a non-slip finish with beveled edges in the horizontal corners to receive the finishing. The metal sheet continues, structurally suspended by 16 cables distributed circularly along the length of the interior perimeter and the exterior of the stair (fig. 16).

We found two types of handrails: a first follows the spiral path in the exterior perimeter and in the eye of the stair, and a second that has a horizonta path and rotates around the circle that produces the stair with the ground from the upper lobby. Despite their geometric differences, we can describe both the following way: a handrail $90 \mathrm{~cm}$ tall formed by a top metal rail, with a rectangular cross section and of dark gray color, and white vertical posts repeated every $60^{\circ}$. The space that delimits the posts and handrail is completed by darkened glass found anchored by four pieces that are crimped to the vertical posts and resist torque. In the area of the stair, the class follows a spiral curve while in the upper zone adjusts to the circular curve (figs. 17 to 20 ).

In the outer perimeter of the staircase we find a total of ten cables, one each $30^{\circ}$. These have different lengths and go from the folded sheet to the interior perimeter of the opening of the upper level. In the eye of the staircase we find 6 cables every $60^{\circ}$ that are aligned in plan with the exterior cables and go from the folded sheet up to the roof of the upper lobby.

Arne Jacobsen wanted to highlight the functionality of each of the stair elements with color. For this reason, the cables are painted red, the folded sheet and the vertical posts are white and the handrails are dark gray. His condition as architect and engineer and industrial engineer led him to employ and define the use of industrial components and the technological characterization of his design and its formal determination. Jacobsen identified with the influence of these new techniques in architectonic form, favoring the proposals and promoting the technological culture applied to architectural elements (Habermann, 2003 and Pracht, 1991).

\section{STRUCTURE}

To understand the structural behavio of the stair, one must first understand the structural behavior of the portion of the building where it is suspended. The lobby is structurally designed through the central bay 8 meters wide bounded by two rows of pillars separated every $4 \mathrm{~m}$. The stair is situated in the middle of the central bay and at one side of the lobby. Its connection with the upper level, upper slab of reinforced concrete and deep beams, generates an opening that relates the two spaces, and structurally speaking is topped with a round hoop of metal that connects monolithically with the rest of the slab.

The stair is generated by a welded steel plate $2 \mathrm{~cm}$ thick with 32 folds. This sheet receives the weight of all the parts and finishes of the handrails. The sum of these loads is proportionally transmitted to the 16 steel cables, $24 \mathrm{~mm}$ in diameter that distribute the tension to the building slabs. As we have said, ten cables every $30^{\circ}$ on the circular exterior perimeter and six cables every $60^{\circ}$ around the eye of the staircase. Each cable has a different length as the exterior cables run from the steel sheet to the circular ring in the floor of the upper lobby and the interior cables run from the steel plate to the ceiling of the upper lobby. The vertical deformation of each of these bars, which ends in a screw, can be regulated through each of the connectors (fig. 21)

One must consider that in the lower part of the second stair we find two supports that connect with the floor of the entrance lobby to cancel out vibrations upon going up or down. We would like to point out that in 1961 Jacobsen designed a linear stair completely suspended by 8 cables in the lobby of the National Bank of Denmark and in this case there was no contact with the ground. Therefore, we can observe that Arne Jacobsen tested technical methods in each of his designs and he perfected them throughout his professional career (AA.vV., 1961; Faber, 1964 y Pracht, 1991).

\section{CONCLUSIONS}

In this work, we faithfully reflected the sparse original documentation we could obtain. And from this, we elaborated a three-dimensional model and we presented ample documentation with which we can highlight different elements of the study.

With this analysis, any architect interested in Arne Jacobsen's spiral staircase can obtain information previously unobtainable until this article becomes clearly available to the public.

We can also conclude that, historically, this is the first suspended spiral staircase with 16 cables and using a folded steel plate as a unifying element. Until this moment, the most common structural design for hanging these elements consisted of suspending each of the steps making up the system or use perimeter stringers that performed the same function. After the spiral stair of the Hotel Royal SAS we found various spiral staircases with similar technical and construction features confirming the technical repercussions had by Jacobsen's stair.

Similarly, in the project that contains the stair, an entrance element of the building is also suspended. With this, we emphasize that the stair design impacts the lobby design on a compositional level and that the decision to hang it is consistent with another element in the same space, thus highlighting the duality as the order and motive of the design.

Finally, after a detailed bibliographical search, we can say that this article is the first document that publicly shows the functional, formal and structural analysis of the Hotel Royal sAs spiral staircase. With this work, we have opened a new source of ideas available to architects or professionals of other areas on the technique and philosophy of Arne Jacobsen.

Notes

1 The building was built in the city of Warren, Michigan between 1949 and 1955 and was inaugurated with the presence of US president, Dwight D. Eisenhower, in 1956 2 The pavillion was designed by the Canadian architect Harold Semmens and Douglas Simpson, and once finished the exposition was sold and reinstalled in the Beligian city of Genk, where today it serves as an elementary school.
ALBERT SAMPER | Architect, Universidad Politècnica de Catalunya (UPC), 2008; Master of Theory and Practice of the Architecture Project, UPC, 2011 and Doctor, Ph.D. Universidad Rovira i Virgili, 2014. His central line of work consists of the geometric analysis of architectural elements. He currently teaches Geometry in the Area of Architectural Graphic Expression in the Universidad Rovira i Virgili. His main fields of interest are fractal geometry and the application of geometry in architecture.
BLAS HERRERA | Mathematician and Geometer, Universidad de Barcelona, 1991; Master of Mathematics, Universidad Autónoma de Barcelona (UAB), 1993 and Doctor of Science, Sc.D., UAB, 1994. His central line of work is geometry and its applied digitalization. He is currently a professor of Applied Mathematics at the Escuela Técnica Superior de Arquitectura of the Universidad Rovira i Virgili. His main fields of interest are classical and differential geometry applied to architecture, the mechanics of fluids and engineering. 